\title{
Effect of a low pressure low temperature hydrogen plasma on the work function of europium
}

\author{
S. Cristofaro, 1,2, a) R. Friedl, ${ }^{2}$ and U. Fantz ${ }^{1,2}$ \\ 1) Max-Planck-Institut für Plasmaphysik, Boltzmannstr. 2, 85748 Garching, Germany \\ 2) AG Experimentelle Plasmaphysik, Universität Augsburg, 86135 Augsburg, Germany
}

(Dated: July 2020)

Europium is a ductile rare earth element and has the lowest function among lanthanides (2.5 eV). Due to its low work function reachable without an activation procedure, Eu is a potential electron emitter. For its application to negative hydrogen ion sources for fusion, the work function of a Eu sample exposed to a low pressure low temperature hydrogen plasma is investigated here. First, a work function of $2.6 \pm 0.1 \mathrm{eV}$ is achieved in the present vacuum conditions (ca. $10^{-6}$ mbar) by heating the sample up to $350^{\circ} \mathrm{C}$. A short hydrogen plasma pulse of 1 min allows to achieve the same low work function, however a longer plasma exposure leads to an increase of the work function, which must be counteracted by heating the sample up to at least $400^{\circ} \mathrm{C}$ to maintain the low work function during plasma. Furthermore, the exposure to hydrogen plasma affects the reactivity of Eu to oxidation when it is exposed to air, showing evidence of a faster oxidation in the areas previously in contact with the plasma. In conclusion, due to the strong reaction to hydrogen plasma and the rapid oxidation in air, Eu is not suitable for application in negative ion sources for fusion.

Keywords: europium, low temperature low pressure plasma, hydrogen plasma

\section{INTRODUCTION}

Rare-earth elements have an important role in a wide range of applications, from optical devices to electronics and catalysts, due to their magnetic, luminescent and electrochemical properties. Among these materials, europium attracts considerable interest. Divalent and trivalent europium is used in optoelectronic devices, as diodes and lasers, due to its phosphorescence properties ${ }^{1}$ and as neutron absorber because of its large neutron capture cross-section $^{2}$. As a rare-earth metal, Eu easily reacts with air and with hydrogen ${ }^{3}$, and europium hydrides are studied to deepen the knowledge on metal hydrides for the search for new hydrogen storage materials ${ }^{45}$. Elemental europium has the lowest work function $\left(2.5 \mathrm{eV}^{\sqrt{6}}\right)$ among lanthanides, hence it might be considered in a wide range of applications where a high electron emission ability is required. In contrast to other electron emitting materials usually applied as hot cathodes and which require temperatures above $1000{ }^{\circ} \mathrm{C}$, Eu does not need any activation procedure allowing for the application of this material also at lower temperatures. For example, negative ion sources for fusion rely on the surface conversion of hydrogen particles into negative ions by electron transfer from a low work function surface ${ }^{7}$. The impinging particles are positive ions and atoms created in a low pressure low temperature hydrogen plasma with typical electron density $10^{16}-10^{17} \mathrm{~m}^{-3}$ and electron temperature of $1-2 \mathrm{eV}$. The fluxes of impinging positive ions and atoms are on the order of $10^{20}-10^{21}$ and $10^{22} \mathrm{~m}^{-2} \mathrm{~s}^{-1}$, respectively. The flux of VUV photons is expected to be on the same order of magnitude as the positive ion flux. The source operates with a background pressure

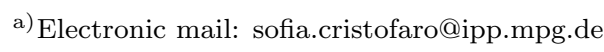

of $10^{-7}-10^{-6}$ mbar and a gas pressure of $0.3 \mathrm{~Pa}$, with plasma pulses of at least one hour. Currently, caesium is evaporated into the source to reduce the surface work function and increase the negative ion yield. However, different low work function materials can be considered for application in negative ion sources to avoid the strong dynamics observed with evaporation of $\mathrm{C} \$ 9$.

In this paper, the work function of elemental europium exposed to hydrogen plasma is studied. Previous investigations of the work function of a thin film $(20-50 \mathrm{~nm})$ of $\mathrm{Eu}$ in $\mathrm{H}_{2}$ gas (up to $30 \mathrm{~Pa}$ ) in a UHV system at room temperature are presented in Knor et al! $\frac{10}{\text {, which shows }}$ that the presence of hydrogen on the surface leads to an increase of the work function of $0.02 \mathrm{eV}$ with respect to the pure metal film when hydrogen is adsorbed and a decrease of $0.02 \mathrm{eV}$ with respect to pure Eu when hydrogen is diffusing in the subsurface. The effects of the exposure to a $\mathrm{H}_{2}$ plasma on the surface work function are instead unknown and are for the first time investigated here.

\section{THE EXPERIMENT}

\section{A. Setup}

The laboratory experiment ACCesS11112 (Augsburg Comprehensive Cesium Setup) consists of a cylindrical stainless steel vessel with a diameter of $15 \mathrm{~cm}$ and a height of $10 \mathrm{~cm}$, as shown in figure 1. The background pressure within the vessel is on the order of $10^{-6}$ mbar. For the plasma phases the chamber is filled with hydrogen gas at a pressure of $10 \mathrm{~Pa}$, and the plasma is generated via inductive RF coupling (frequency of $27.12 \mathrm{MHz}$, maximal RF power of $600 \mathrm{~W}$ ) using a planar coil located on top of the vessel. The europium sample $\left(30 \times 30 \mathrm{~mm}^{2}\right.$, thickness $1 \mathrm{~mm}$, purity $99.9 \%$, with a rough surface) was 


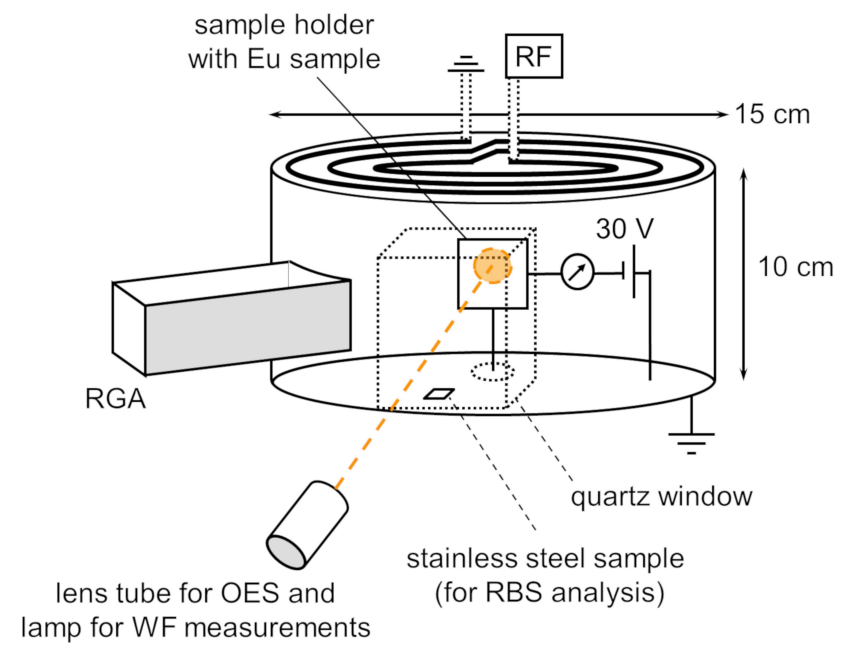

Figure 1. Sketch of the experimental setup ACCesS with the different diagnostics applied for the current investigations.

purchased from the supplier company abcr ${ }^{13}$ and was stored in a sealed can in dry Ar atmosphere to prevent any oxidation. The sample can be clamped to a sample holder, which is electrically and thermally insulated from the vessel and located near the center of the experimental chamber. The surface temperature of the front side of the sample is constantly monitored by means of a thermocouple, and the sample can be heated up to $350^{\circ} \mathrm{C}$ in vacuum and $400{ }^{\circ} \mathrm{C}$ in plasma by means of a coaxial heating wire within the sample holder. During plasma operation the temperature of the sample inevitably increases up to $200^{\circ} \mathrm{C}$.

A residual gas analyzer (RGA) is used to monitor the background gases during operation. During plasma operation optical emission spectroscopy (OES) is applied for monitoring the plasma parameters as described in Friedl et al.14. The possible release of europium from the sample is investigated after the performed investigations by Rutherford backscattering spectroscopy $\frac{15}{15 B S}$ ) on a small stainless steel sample $\left(10 \times 10 \mathrm{~mm}^{2}\right)$ laying on bottom of the vessel in front of the sample.

The work function (WF) of the sample is evaluated considering the photoelectric effect induced by irradiation and applying the Fowler method $\frac{16}{16}$. The setup and the analysis are described in detail in Fried ${ }^{117}$ with the improvements presented in Cristofaro et al ${ }^{12}$, and here it is shortly summarized. A high pressure mercury lamp $(100 \mathrm{~W})$ is applied as a broadband light source, and the light passes through an interference filter and is focused on the sample holder resulting in a spot diameter of about $1.5 \mathrm{~cm}$. The photoemitted electrons are collected at the vessel walls by applying a bias voltage of $-30 \mathrm{~V}$ to the sample against the grounded walls. The photocurrents are measured by a Keithley 602 Electrometer, and the work function is absolutely determined by measuring the photocurrents for different interference filters. Twenty filters with central transmission wavelengths between 239 and $852 \mathrm{~nm}$ and with a nominal FWHM of $10 \mathrm{~nm}$ are available for the current investigations, making accessible the mean photon energies in the range between 5.04 and $1.45 \mathrm{eV}$. The entire setup was calibrated by means of an absolutely calibrated spectrometer and a radiant power meter. The photocurrents cannot be measured during plasma operation, since the plasma electrons will contribute to the measured current and it will not be possible to discern the low photocurrents from the total current. Hence, the work function is measured within the first minutes (max. $3 \mathrm{~min}$ ) after switching off the plasma. The plasma is thus pulsed with length ranging from few seconds up to several hours. For each work function evaluation the photocurrents of at least five filters are measured, and the interference filters are exchanged appropriately depending on the work function in order to fulfill the validity conditions 17 . The photocurrents could always be nicely fit by a Fowler curve, and the typical error of the fitted work function value is about $0.1 \mathrm{eV}$.

\section{B. Relevant species and fluxes}

The work function can be strongly influenced by the interaction between the surface and the particles from the background gas and the plasma, since adsorption, erosion, sputtering and chemical reactions can occur. ACCesS has a limited vacuum level, and the background gas is mostly composed by water vapor, as observed with RGA. The flux of impurities towards the surface is far from being negligible (around $10^{18} \mathrm{~m}^{-2} \mathrm{~s}^{-1}$ ) and can lead to formation of oxides and hydroxyl species.

During the plasma campaigns hydrogen is introduced in the experiment reaching a pressure of $10 \mathrm{~Pa}$ for the whole day. During the gas phase, molecular hydrogen at room temperature can deposit onto the surface and the flux of $\mathrm{H}_{2}$ molecules is around $10^{24} \mathrm{~m}^{-2} \mathrm{~s}^{-1}$.

For the plasma phases, an RF power of $250 \mathrm{~W}$ is applied resulting in a plasma with typical electron temperature and density of $2 \mathrm{eV}$ and $1.4 \times 10^{16} \mathrm{~m}^{-3}$, respectively. In this phase, atomic and molecular hydrogen, positive hydrogen ions $\left(\mathrm{H}^{+}, \mathrm{H}_{2}^{+}, \mathrm{H}_{3}^{+}\right)$and UV/VUV radiation emitted by de-excitation of plasma particles can contribute to surface modifications. Typical fluxes of atomic hydrogen and positive ions (at $10 \mathrm{~Pa}$ the dominant positively charged species is $\mathrm{H}_{3}^{+}$) are approximately $10^{22} \mathrm{~m}^{-2} \mathrm{~s}^{-1}$ and $10^{20} \mathrm{~m}^{-2} \mathrm{~s}^{-1}$, respectively. The temperature of the atoms is assumed equal to the gas temperature which is measured around $0.05 \mathrm{eV}$ by OES. The positive ions have energies of $8 \mathrm{eV}$ in maximum determined by the potential drop at the surface, which is not biased during plasma. The flux of VUV photons towards the surface (with energies between 3 and $15 \mathrm{eV}$ ) is between $10^{19}-10^{20} \mathrm{~m}^{-2} \mathrm{~s}^{-1}$ as determined from recent measurements 18 .

The relevant fluxes towards the surface during vacuum, gas and plasma phases at ACCesS are summarized in table I 
Table I. Relevant species and corresponding fluxes towards the surface in vacuum, gas and plasma conditions.

\begin{tabular}{l|l|lc}
\hline \multicolumn{4}{c}{ Typical fluxes in $\left[\mathrm{m}^{-2} \mathrm{~s}^{-1}\right]$ during } \\
vacuum & gas & plasma \\
\hline Residual gases (water vapor): $10^{18}$ & Molecular hydrogen: $10^{24}$ & $\begin{array}{c}\text { Atomic hydrogen: } \\
\text { Positive ions: }\end{array}$ & $10^{22}$ \\
& & VUV photons: & $10^{19}-10^{20}$ \\
\hline
\end{tabular}

\section{Europium chemistry}

Europium is one of the most reactive element due to its half-filled electron shell, reacting easily with water and oxygen. Eu reacts with moist air to form $\mathrm{Eu}(\mathrm{OH})_{2} \cdot \mathrm{H}_{2} \mathrm{O}^{19}$ (characterized by a yellow color), which gradually deteriorates forming the white compound $\mathrm{Eu}(\mathrm{OH})_{3}$ also in inert atmosphere ${ }^{20}$ (formation enthalpy of $\left.-13.9 \mathrm{eV}^{19}\right)$. From Rau et al! ${ }^{21}$, the latter thermally decomposes in air atmosphere in $\mathrm{EuOOH}$ in a temperature range between $225^{\circ} \mathrm{C}$ and $300^{\circ} \mathrm{C}$ followed by a decomposition into the white compound $\mathrm{Eu}_{2} \mathrm{O}_{3}$ between $435^{\circ} \mathrm{C}$ and $465^{\circ} \mathrm{C}$, while the process may be different in a vacuum environment. Consequently, it can be expected that $\mathrm{Eu}$ compounds are forming on the surface of the sample during the transport from the sealed can to the ACCesS experiment, before going into vacuum. Additionally, the main component of the residual gases in the experiment vacuum is water vapor, and formation of Eu hydroxides cannot be excluded even in vacuum conditions. The bond dissociation energy between $\mathrm{Eu}$ and $\mathrm{O}$ is of $4.9 \mathrm{eV}^{22}$.

When hydrogen gas is injected into the experiment, molecular hydrogen can deposit onto the surface forming $\mathrm{EuH}_{2}$ (which is a dark semiconductor ${ }^{23}$ ). Formation of $\mathrm{EuH}_{2}$ was observed by direct reaction between hydrogen and $\mathrm{Eu}$ at $400{ }^{\circ} \mathrm{C}$ with very high $\mathrm{H}_{2}$ pressures (above $10^{5} \mathrm{~Pa}$ ) in Bischof et al. ${ }^{24}$ and Kohlmann et al. $\stackrel{4}{\text {. Forma- }}$ tion of $\mathrm{EuH}_{2}$ at lower pressures (between 20 and $700 \mathrm{~Pa}$ ) was observed in Schoenes et al! 25 , where a $\mathrm{EuH}_{2}$ film was created by pulsed laser ablation of Eu hit by a high power pulsed laser in hydrogen atmosphere.

When the plasma is switched on, hydrogen atoms, molecules and positive ions and photons can hit the surface, and several interactions can take place with the surface, like ad-/absorption, erosion, sputtering and chemical reactions. Furthermore, the plasma heats the surface, hence thermal effects take place simultaneously. In this phase, hydrogen can be absorbed and diffused in the bulk leading to the formation of compounds at and below the surface. In addition to $\mathrm{EuH}_{2}$, formation of $\mathrm{EuH}_{3}$, which is an insulator, cannot be excluded. However, this compound has been observed only in very high hydrogen pressure (around GPa) experiments $26 \mid 27$, and its synthesis is very difficult and has been achieved just in recent years 26 .

The presence of compounds on the sample surface and their stochiometric composition unfortunately cannot be assessed since they would require in-situ techniques not available at ACCesS. Nevertheless, the presence of these compounds can affect the work function of the surface, which is investigated here.

\section{RESULTS AND DISCUSSION}

\section{A. Work function in vacuum and gas phase}

To install the europium sample in the experiment, the sample was exposed to air for maximum 10 minutes. No evidence of oxidation was discernible by eye, nevertheless the formation of hydroxides on the sample surface cannot be excluded and avoided. A first measurement of the work function in vacuum shows that the work function is higher than $4.5 \mathrm{eV}$ indicating that there are impurities on the surface. In order to decompose and possibly remove the impurities, the sample is heated in vacuum conditions.

Figure 2 shows the temperature $T_{\mathrm{s}}$ and the work function $\chi$ of the europium sample during the heating of the sample. The temperature is step-wise increased from room temperature up to $350{ }^{\circ} \mathrm{C}$. The work function initially is higher than $4.5 \mathrm{eV}$ until the temperature reaches $250{ }^{\circ} \mathrm{C}$, then the work function starts to decrease. At

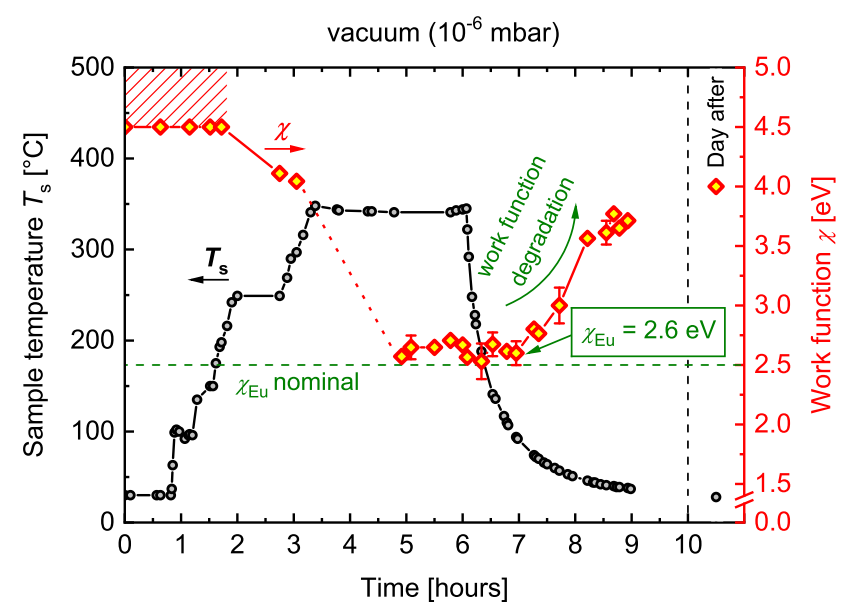

Figure 2. Work function of $\mathrm{Eu}$ in vacuum with increasing sample temperature. Once the temperature reaches $350^{\circ} \mathrm{C}$, the measured work function is $2.6 \pm 0.1 \mathrm{eV}$, close to the nominal value. 
$350{ }^{\circ} \mathrm{C}$ the work function reaches the stable value of $2.6 \pm 0.1 \mathrm{eV}$ for more than one hour, consistent within the error bar with the work function of pure Eu. During the heating of the sample, RGA signals show two peaks in the water content at $200^{\circ} \mathrm{C}$ and at $250^{\circ} \mathrm{C}$, and the background pressure shows peaks from $9 \times 10^{-6} \mathrm{mbar}$ up to $3.3 \times 10^{-5}$ mbar every time the temperature increases. The pressure then stabilizes at $6 \times 10^{-6} \mathrm{mbar}$ once the surface reaches $350{ }^{\circ} \mathrm{C}$. The peaks in the background pressure and on RGA suggest a cleaning of the surface with desorption of particles, revealing the underlying pure material and allowing to achieve the low work function.

Figure 2 shows also the subsequent cooling down of the sample once the heating is stopped after 6 hours of operation. The temperature decreases continuously and the work function does not immediately change, but it is stable at $2.6 \mathrm{eV}$ for 30 minutes. After half an hour and with a surface temperature below $100^{\circ} \mathrm{C}$, the work function starts to increase with a degradation rate of $+0.8 \mathrm{eV} / \mathrm{h}$ and saturates at $4.0 \mathrm{eV}$ over a night in vacuum. A similar degradation is observed also when $\mathrm{H}_{2}$ gas is introduced at a pressure of $10 \mathrm{~Pa}$ after the heating of the sample in vacuum: the work function increases from $2.6 \mathrm{eV}$ to $3.8 \mathrm{eV}$ over one hour and 20 minutes, with a degradation rate below $0.9 \mathrm{eV} / \mathrm{h}$. It is thus concluded that the deterioration of the work function is due to the adsorption of residual gases re-depositing on the low work function surface, and it is not influenced on the possible formation of europium hydrides and hydrogen adsorption on the surface. After the degradation, the low work function can be retrieved by heating the surface again to $350^{\circ} \mathrm{C}$.

\section{B. Work function during plasma exposure}

In order to monitor the work function during plasma, a pulsed hydrogen plasma at $10 \mathrm{~Pa}$ and $250 \mathrm{~W}$ is applied without active heating of the sample. Figure 3 shows the work function of the europium sample after each pulse (depicted in grey in the figure) starting from an initial work function of $3.8 \mathrm{eV}$. The temperature reached by the sample during and after the plasma pulses is also plotted.

First, a very short plasma pulse of 1 minute is loaded, and a work function of $2.6 \mathrm{eV}$ is achieved even if the surface temperature does not exceed $75^{\circ} \mathrm{C}$. The plasma is thus able to retrieve the low work function, as alternative to the heating procedure, indicating that the plasma particles and photons hitting the surface have sufficient energy to break the bonds between europium and background impurities.

After the first pulse subsequent longer hydrogen plasma pulses are applied, and a gradual increase of the work function is observed. After 30 minutes of overall plasma exposure with a maximal sample temperature of $20{ }^{\circ} \mathrm{C}$, the work function increases from 2.6 to $3.5 \mathrm{eV}$ with a degradation rate in plasma of $1.8 \mathrm{eV} / \mathrm{h}$. The degradation rate then decreases, and a work function of

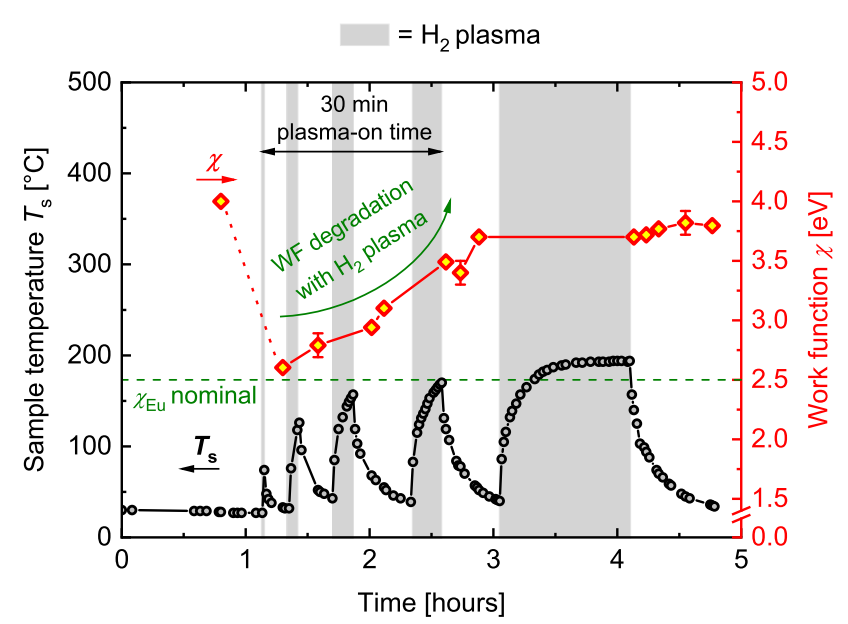

Figure 3. Sample temperature and work function of Eu during exposure to $\mathrm{H}_{2}$ plasma pulses at $10 \mathrm{~Pa}$ and $250 \mathrm{~W}$ without active heating of the sample.

$3.7-3.8 \mathrm{eV}$ is measured after 1 hour of total plasma exposure. The work function saturates around $4.2 \mathrm{eV}$ for longer plasma-on time ( $\sim 3$ hours).

This degradation occurring during plasma is different from the degradation observed during vacuum and gas phases, because in the latter case a short plasma pulse is able to retrieve the low work function, while in the case of the "plasma degradation" low work functions cannot be recovered with short plasma pulses. This plasma degradation may be due to damages to the surface due to energetic particles and photons, or to the adsorption, or even absorption and diffusion, of atomic or molecular hydrogen or of positive ions neutralizing at the surface.

In order to retrieve the low work function after the plasma degradation, the sample is heated again to a temperature of $350^{\circ} \mathrm{C}$ in vacuum, similarly to the campaign of figure 2. During the heating of the sample no variation of the RGA signals is observed, however the background pressure shows some peaks from $8 \times 10^{-6}$ mbar up to $1.3 \times 10^{-5}$ mbar every time the temperature increases, stabilizing at $7 \times 10^{-6}$ mbar once the surface reaches $350{ }^{\circ} \mathrm{C}$. This suggests that particles are released from the surface during the heating, though it is not possible to detect the particle species because of the low sensitivity of the residual gas analyzer. Finally, a work function of $2.6 \mathrm{eV}$ is successfully achieved again.

\section{Work function during plasma exposure with active heating}

In order to counteract the increase of the work function during plasma, the sample is simultaneously heated in the campaign shown in figure 4. The surface was exposed to plasma for a long time before the campaign, and the surface work function is saturated at around $4.2 \mathrm{eV}$, 


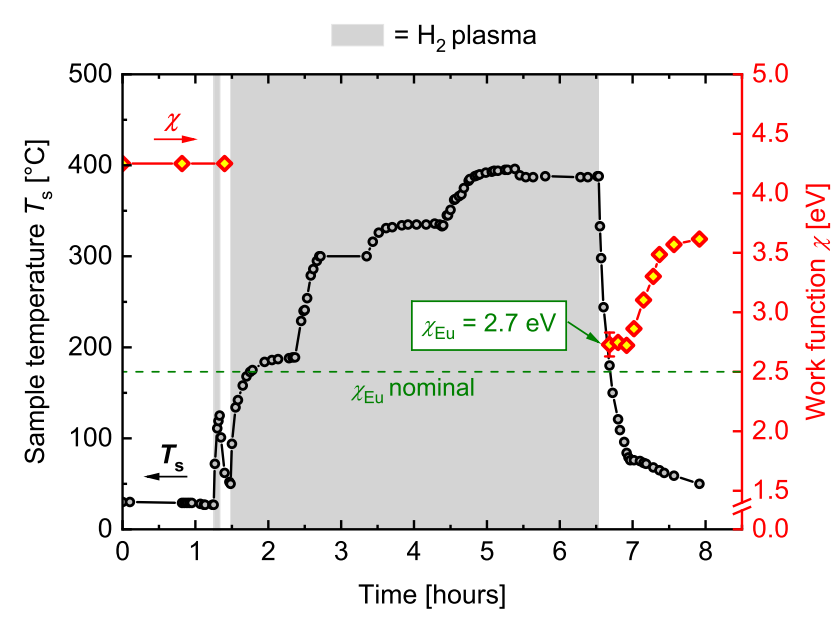

Figure 4. Sample temperature and work function of europium due to exposure to a short $(5 \mathrm{~min})$ and a long (5 hours) $\mathrm{H}_{2}$ plasma pulse at $10 \mathrm{~Pa}$ and $250 \mathrm{~W}$. During the second pulse the sample is additionally heated up to $400{ }^{\circ} \mathrm{C}$.

as proved by the still high work function measured after the first short plasma pulse of $5 \mathrm{~min}$ in figure 4. The temperature of the sample is then increased during a long plasma exposure (ca. 5 hours) up to $400^{\circ} \mathrm{C}$, which is the maximum temperature that the sample can reach in the current setup. After the long plasma treatment a work function of $2.7 \mathrm{eV}$ is observed, close to the lowest value previously measured. A dedicated campaign with plasma and active heating but with a lower surface temperature $\left(325^{\circ} \mathrm{C}\right)$ revealed a higher work function equal to $3.3 \mathrm{eV}$. Therefore, a minimum temperature of $400^{\circ} \mathrm{C}$ is required to maintain a low work function during plasma operation. This temperature is higher with respect to the case in vacuum, where a temperature of $350{ }^{\circ} \mathrm{C}$ is enough to achieve $2.6 \mathrm{eV}$. This is attributed to the higher fluxes and different particles species and, hence, to an enhanced degradation during the plasma phase.
In figure 4 the work function is monitored also after plasma, and it increases from 2.7 to $3.6 \mathrm{eV}$ with a degradation rate of $1.5 \mathrm{eV} / \mathrm{h}$, higher than the degradation rate measured in vacuum or gas phase after the heating campaign before any plasma treatment, indicating a higher reactivity of the surface to the residual gases after the exposure to the hydrogen plasma. The reason of such different behavior cannot be assessed, and modifications of the surface and subsurface due to hydrogen absorption or diffusion with formation of highly reactive europium hydrides cannot be excluded.

\section{Ex-situ analysis and sample air exposure}

A visible change of the sample surface was not observed during the heating and plasma campaigns. The sample has shown a good resistance and mechanical stability in vacuum with temperatures up to $400^{\circ} \mathrm{C}$ and in plasma environment. An RBS analysis of the stainless steel sample that was placed on the bottom of the experiment showed that no Eu was deposited on the sample, meaning that no sputtering or erosion of Eu occurred.

After the campaigns the experiment was vented and the sample was exposed to air immediately. A fast and irreversible oxidation of the surface occurred, and white structures appeared on the surface within 15 minutes. In particular, the portion of the surface exposed to plasma during the investigations was oxidizing much faster with respect to the sides of the sample, which were covered by the clamps during the campaigns. This fast oxidation suggests the presence of europium hydride $\mathrm{EuH}_{2}$, which rapidly oxidizes in air ${ }^{28}$ forming hydroxide characterized by a white color. In order to preserve the Eu sample after the current investigations, the sample was immediately stored in a separate vacuum chamber connected to a small pump stand (with a background pressure of $10^{-6}$ mbar) and no surface analysis was possible on the sample. Pictures of the sample in the different stages are shown in figure 5 .

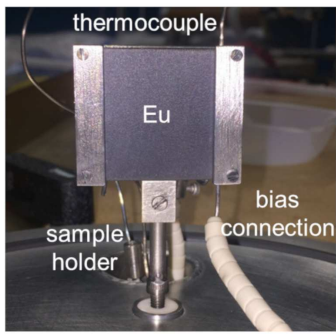

before investigations

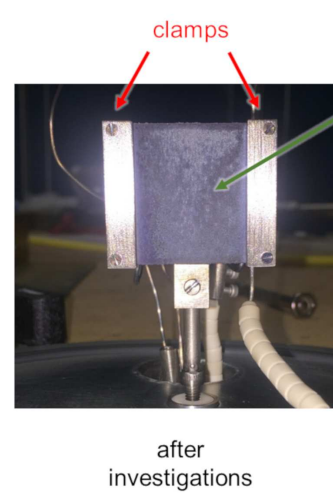

investigations

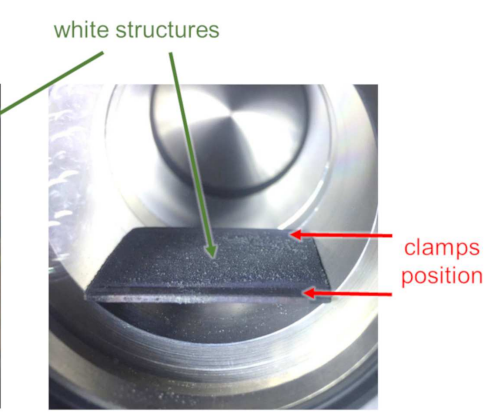

separate vacuum chamber

Figure 5. Pictures of the Eu sample before and after the investigations and during the subsequent storage in a separate vacuum chamber. Clear evidence of oxidation of the sample area previously in contact with the plasma is observed as white color structures developing on the surface between the clamps, while the region below the clamps is less affected. 


\section{SUMMARY AND CONCLUSIONS}

Europium owns the lowest work function among lanthanides $(2.5 \mathrm{eV})$, making this material interesting for applications that require low work function surfaces, as for example negative ion sources. The work function of a sample of europium is thus studied at the laboratory experiment ACCesS in a moderate vacuum (ca. $10^{-6}$ mbar) and during hydrogen plasma exposure. In these vacuum conditions the sample needs to be heated to temperatures up to $350^{\circ} \mathrm{C}$ in order to achieve and maintain a work function of $2.6 \pm 0.1 \mathrm{eV}$. Once the heating is stopped, the work function shows a clear degradation of $+0.8 \mathrm{eV} / \mathrm{h}$ due to the influence of the background gases. The low work function can be easily retrieved by heating the sample again to $350{ }^{\circ} \mathrm{C}$ or with a short plasma pulse $(1 \mathrm{~min})$. However, a longer exposure to the hydrogen plasma is found to affect strongly the work function of the sample: a degradation caused by plasma at a rate of $+1.8 \mathrm{eV} / \mathrm{h}$ is observed, and the work function saturates at $4.2 \mathrm{eV}$. To counteract this degradation it is necessary to heat the sample simultaneously at least up to $400^{\circ} \mathrm{C}$, allowing to achieve work function below $2.7 \mathrm{eV}$ also during long plasma operation. The faster oxidation of the europium sample after the ventilation of the experiment suggests a high reactivity of this material to hydrogen plasmas and hydrogen absorption. At the ion sources higher particles and photon fluxes can be achieved with respect to $\mathrm{AC}$ CesS, and this can lead to a faster degradation of the work function and to the need of higher temperatures to maintain the low work function. Additionally, ion sources for fusion need venting for maintenance, hence it would be required to completely exchange all Eu surfaces at each ventilation. Consequently, $\mathrm{Eu}$ is not suitable for application at ion sources due to its strong reactivity.

\section{ACKNOWLEDGMENTS}

This work has been carried out within the framework of the EUROfusion Consortium and has received funding from the Euratom research and training programme 2014-2018 and 2019-2020 under grant agreement No 633053. The views and opinions expressed herein do not necessarily reflect those of the European Commission.

\section{REFERENCES}

${ }^{1}$ J. Lucas, P. Lucas, T. L. Mercier, et al., "Chapter 15 - Introduction to Rare Earth Luminescent Materials," in Rare Earths (Elsevier, Amsterdam, 2015) p. 251.

${ }^{2}$ G. Leinweber, D. P. Barry, J. A. Burke, et al., "Europium resonance parameters from neutron capture and transmission measurements in the energy range 0.01-200 eV," Ann. Nucl. Energy 69, $74(2014)$
${ }^{3}$ A. Simon, "Oxidation by Hydrogen in the Chemistry and Physics of the Rare-Earth Metals," Angew. Chem. Int. Ed. 51, 4280 (2012)

${ }^{4}$ H. Kohlmann and K. Yvon, "The crystal structures of $\mathrm{EuH}_{2}$ and $\mathrm{EuLiH}_{3}$ by neutron powder diffraction," J. Alloys Compd. 299, L16 (2000).

${ }^{5}$ H. Kohlmann, "Solid-State Structures and Properties of Europium and Samarium Hydrides," Eur. J. Inorg. Chem. 2010, $2582(2010)$

${ }^{6}$ H. B. Michaelson, "The work function of the elements and its periodicity," J. Appl. Phys. 48, 4729 (1977)

${ }^{7}$ M. Bacal and M. Wada, "Negative hydrogen ion production mechanisms," Appl. Phys. Rev. 2, 021305 (2015)

${ }^{8}$ B. Heinemann, U. Fantz, W. Kraus, et al., "Towards large and powerful radio frequency driven negative ion sources for fusion," New J. Phys. 19, 015001 (2017).

${ }^{9}$ C. Wimmer, A. Mimo, M. Lindauer, U. Fantz, and the NNBI Team, "Improved understanding of the Cs dynamics in large $\mathrm{H}^{-}$sources by combining TDLAS measurements and modeling," AIP Conf. Proc. 2011, 060001 (2018).

${ }^{10}$ M. Knor, R. Nowakowski, and R. Duś, "Surface and bulk phenomena in the process of hydride formation in thin films of rare earth metals: Comparison between terbium and europium," Appl. Surf. Sci. 257, 8241 (2011)

${ }^{11} \mathrm{R}$. Friedl and U. Fantz, "Influence of $\mathrm{H}_{2}$ and $\mathrm{D}_{2}$ plasmas on the work function of caesiated materials," J. Appl. Phys. 122, 083304 (2017)

${ }^{12}$ S. Cristofaro, R. Friedl, and U. Fantz, "Correlation of Cs flux and work function of a converter surface during long plasma exposure for negative ion sources in view of ITER," Plasma Res. Express (2020), 10.1088/2516-1067/abae81

13 abcr GmbH, Germany, 2016, https://www.abcr.de/

${ }^{14}$ R. Friedl and U. Fantz, "Influence of cesium on the plasma parameters in front of the plasma grid in sources for negative hydrogen ions," AIP Conf. Proc. 1515, 255 (2013)

${ }^{15}$ W. Reiber, Department of Experimental Physics IV, University of Augsburg, 2017.

${ }^{16}$ R. H. Fowler, "The Analysis of Photoelectric Sensitivity Curves for Clean Metals at Various Temperatures," Phys. Rev. 38, 45 (1931)

${ }^{17}$ R. Friedl, "Enhancing the accuracy of the Fowler method for monitoring non-constant work functions," Rev. Sci. Instrum. 87, 043901 (2016)

${ }^{18}$ C. Fröhler-Bachus, Private communication, Department of Experimental Plasma Physics, University of Augsburg, 2020.

${ }^{19}$ J. A. Rard, "Chemistry and thermodynamics of europium and some of its simpler inorganic compounds and aqueous species," Chem. Rev. 85, 555 (1985)

${ }^{20} \mathrm{D}$. A. Johnson, Recent Advances in the Chemistry of the LessCommon Oxidation States of the Lanthanide Elements, edited by H. J. Emeléus and A. G. Sharpe, Advances in Inorganic Chemistry and Radiochemistry, Vol. 20 (Academic Press, 1977).

${ }^{21}$ R. C. Rau and W. J. Glover, "Thermal Decomposition of Europium Hydroxide," J. Am. Ceram. Soc. 47, 382 (1964)

${ }^{22}$ D. R. Lide, ed., CRC Handbook of Chemistry and Physics (CRC Press, Boca Raton, FL, 2005).

${ }^{23}$ R. Bischof, E. Kaldis, and P. Wachter, "EuH $\mathrm{H}_{2}$ : A new ferromagnetic semiconductor," J. Magn. Magn. Mater. 31-34, 255 (1983)

${ }^{24}$ R. Bischof, E. Kaldis, and P. Wachter, "Synthesis, crystallographic and physical properties of europium dihydride," J. Less Common Met. 111, 139 (1985)

${ }^{25}$ J. Schoenes, H. Schröter, B. Lobbenmeier, et al., "Magnetic, optical and magneto-optical properties of PLD grown $\mathrm{EuH}_{2}$ films," J. Phys. Conf. Ser. 200, 072087 (2010)

${ }^{26}$ T. Matsuoka, H. Fujihisa, N. Hirao, et al., "Structural and Valence Changes of Europium Hydride Induced by Application of High-Pressure $\mathrm{H}_{2}$," Phys. Rev. Lett. 107, 025501 (2011)

${ }^{27}$ H. Saitoh, A. Machida, T. Matsuoka, et al., "Phase diagram of the Eu-H system at high temperatures and high hydrogen pressures," Solid State Commun. 205, 24 (2015)

${ }^{28}$ R. L. Zanowick and W. E. Wallace, "Ferromagnetism in EuH 2 ," Phys. Rev. 126, 537 (1962). 